\title{
Collinear Equilibrium Points in the Relativistic R3BP when the Bigger Primary is a Triaxial Rigid Body
}

\author{
Nakone Bello ${ }^{1, a}$ and Aminu Abubakar Hussain ${ }^{2, b}$ \\ ${ }^{1}$ Department of Mathematics, Faculty of Science, Usmanu Danfodiyo University, Sokoto, Nigeria \\ ${ }^{2}$ Department of Mathematics, Faculty of Science, Ahmadu Bello University, Zaria, Nigeria \\ abnakone@yahoo.com (corresponding author), bdadinduniya@gmail.com
}

Keywords: Celestial Mechanics, Triaxiality, Relativity, R3BP.

\begin{abstract}
This study examines the effect of the relativistic factor as well as the triaxiality effect of the bigger primary on the positions and stability of the collinear points in the frame work of the post-Newtonian approximation. Using semi-analytical and numerical approach the collinear points are found to be unstable. A numerical exploration in this connection, with the Earth-Moon system, reveals that the relativistic factor has an effect on these positions. It is also found that under the combined effect of relativistic factor and triaxiality, the collinear point $L_{1}$ moves towards the primaries with the increase in triaxiality, while $L_{2}$ and $L_{3}$ move away from the bigger primary. It is also seen that in most of the cases in the presence of triaxiality, the effect of relativistic factor on the positions of $L_{1}$ and $L_{3}$ is not observable; however it has an observable effect on the position of $L_{2}$ in the presence of triaxiality except for the case 2 .
\end{abstract}

\section{Introduction}

In the restricted three-body problem (R3BP), two massive bodies of finite masses $m_{1}$ and $m_{2}$ called bigger and smaller primary respectively having spherical symmetry move about their center of mass in circular orbits. A third mass $m_{3}$, the infinitesimal one, moves under the combined gravitational attraction of the two bodies but does not influence their motion. This problem possesses five equilibrium points, three collinear points $L_{1}, L_{2}$ and $L_{3}$ which are in general unstable and two triangular points $L_{4}$ and $L_{5}$ which are stable for the mass ratio $\mu<\mu_{0}=0.038520 \ldots$ Szebehely [1].

The relativistic restricted three-body problem was originally studied by Brumberg [2]. Bhatnagar and Hallan [3] were the first to study the stability of triangular points of the same model problem and found that the triangular point are unstable in the whole region $0 \leq \mu \leq \frac{1}{2}$ contrary to the classical case where they are stable for $\mu<\mu_{0}$ where $\mu$ is the mass ratio and $\mu_{0}=0.038520 \ldots$

Douskos and Perdios [4] reinvestigated the same model problem and found the region of stability of triangular points as $0<\mu<\mu_{0}-\frac{17 \sqrt{69}}{486 c^{2}}$, where $c$ is the dimensionless speed of light and $\mu_{0}=0.038520 \ldots$ is the Routh's value. Later on, Ahmed et al. [5]reexamined the same model problem and found the region of stability of triangular points as $0 \leq \mu<0.03840$.

Ragos et al. [6] studied the existence, position and stability of collinear points in the relativistic R3BP.

In recent years, there has been a strong revival of interest in the relativistic R3BP. Many perturbing forces i.e. radiation, oblateness, perturbations in the centrifugal and Coriolis forces etc. have been included in the study of relativistic R3BP. Several authors (Abd El-Bar and Abd ElSalam [7]; Abd El-Bar and Abd El-Salam [8]; Abd El-Salam and Abd El-Bar [9]; Katour et al. [10]; 
Abd El-Bar et al. [11]) have focused their study on the locations of equilibrium points of relativistic $\mathrm{R} 3 \mathrm{BP}$ under some various aspects of the above mentioned perturbing forces.

Singh and Bello [12-14] have studied the locations and stability of triangular points of the relativistic R3BP under some of the above mentioned perturbing forces.

In general, the celestial bodies are not perfect spheres. They are either oblate or triaxial. From authors' knowledge no work has been attempted yet by any researcher on the locations and stability of the collinear points when the bigger primary is a triaxial rigid body in the relativistic R3BP.

Hence it raised a curiosity in our minds to study the effect of triaxiality of the bigger primary on the location and stability of collinear equilibrium points in the relativistic R3BP.

This paper is organized as follows: In Section 2, the equations governing the motion are presented; Section 3 describes the positions of collinear points, while their linear stability is analyzed in Section 4. A numerical application of these results and discussion are given in Section 5, and Section 6, respectively. Finally, Section 7 conveys the main findings of this paper.

\section{Equations of motion}

The pertinent equations of motion of the infinitesimal mass in the relativistic R3BP when the bigger primary is a triaxial rigid body, in a barycentric synodic coordinate system $(\xi, \eta)$ and dimensionless variables are given by Brumberg [2] and Bhatnagar and Hallan [3] as:

$$
\begin{aligned}
& \ddot{\xi}-2 n \dot{\eta}=\frac{\partial W}{\partial \xi}-\frac{d}{d t}\left(\frac{\partial W}{\partial \dot{\xi}}\right) \\
& \ddot{\eta}+2 n \dot{\xi}=\frac{\partial W}{\partial \eta}-\frac{d}{d t}\left(\frac{\partial W}{\partial \dot{\eta}}\right)
\end{aligned}
$$

where $W$ is the potential-like function of the relativistic R3BP.

As Katour et al. [10] we do not include the triaxiality parameters $\sigma_{i}(i=1,2)$ in the relativistic part of $W$ since the magnitude of these terms is so small due to $c^{-2}$ where $c$ is the speed of light.

$$
\begin{aligned}
& W=\frac{1}{2}\left(1+\frac{3}{2}\left(2 \sigma_{1}-\sigma_{2}\right)\right)\left(\xi^{2}+\eta^{2}\right)+\frac{1-\mu}{\rho_{1}}+\frac{\mu}{\rho_{2}}+\frac{1-\mu}{2 \rho_{1}^{3}}\left(2 \sigma_{1}-\sigma_{2}\right)+\frac{3(1-\mu) \eta^{2}}{2 \rho_{1}^{5}}\left(\sigma_{2}-\sigma_{1}\right) \\
& +\frac{1}{c^{2}}\left[-\frac{3}{2}\left(1-\frac{1}{3} \mu(1-\mu)\right)\left(\xi^{2}+\eta^{2}\right)+\frac{1}{8}\left\{\dot{\xi}^{2}+\dot{\eta}^{2}+2(\xi \dot{\eta}-\eta \dot{\xi})+\xi^{2}+\eta^{2}\right\}^{2}\right. \\
& +\frac{3}{2}\left(\frac{1-\mu}{\rho_{1}}+\frac{\mu}{\rho_{2}}\right)\left(\dot{\xi}^{2}+\dot{\eta}^{2}+2(\xi \dot{\eta}-\eta \dot{\xi})+\xi^{2}+\eta^{2}\right)-\frac{1}{2}\left(\frac{(1-\mu)^{2}}{\rho_{1}^{2}}+\frac{\mu^{2}}{\rho_{2}^{2}}\right)+\mu(1-\mu)\left\{\left(4 \dot{\eta}+\frac{7}{2} \xi\right)\left(\frac{1}{\rho_{1}}-\frac{1}{\rho_{2}}\right)\right. \\
& \left.\left.-\frac{\eta^{2}}{2}\left(\frac{\mu}{\rho_{1}^{3}}+\frac{1-\mu}{\rho_{2}^{3}}\right)+\left(-\frac{1}{\rho_{1} \rho_{2}}+\frac{3 \mu-2}{2 \rho_{1}}+\frac{1-3 \mu}{2 \rho_{2}}\right)\right\}\right]
\end{aligned}
$$

$$
\begin{aligned}
& \rho_{1}^{2}=(\xi+\mu)^{2}+\eta^{2} \\
& \rho_{2}^{2}=(\xi+\mu-1)^{2}+\eta^{2}
\end{aligned}
$$


and $n$, the perturbed mean motion of the primaries is given by

$$
n=1+\frac{3}{4}\left(2 \sigma_{1}-\sigma_{2}\right)-\frac{3}{2 c^{2}}\left(1-\frac{1}{3} \mu(1-\mu)\right)
$$

where $0<\mu \leq \frac{1}{2}$ is the ratio of the mass of the smaller primary to the total mass of the primaries; $\rho_{1}$ and $\rho_{2}$ are distances of the infinitesimal mass from the bigger and smaller primary, respectively. $\sigma_{i}<<1,(i=1,2)$ characterize the triaxiality of the bigger primary are given by $\sigma_{1}=\frac{a^{2}-h^{2}}{5 R^{2}}, \sigma_{2}=\frac{b^{2}-h^{2}}{5 R^{2}}$ (McCuskey [15]) with $a, b, h$ as lengths of its semi-axes.

\section{Locations of collinear points}

Equilibrium points are those points at which no resultant force acts on the third infinitesimal body. Therefore, if it is placed at any of these points with zero velocity, it will stay there. In fact, all derivatives of the coordinates with respect to the time are zero at these points. Therefore, the equilibrium points are solutions of equations

$$
W_{\xi}=0 \text { and } W_{\eta}=0
$$

$W_{\xi}$ and $W_{\eta}$ may be written as

$$
\begin{aligned}
& W_{\xi}=\xi-\frac{(1-\mu)(\xi+\mu)}{\rho_{1}^{3}}-\frac{\mu(\xi-1+\mu)}{\rho_{2}^{3}}+\left(3 \sigma_{1}-\frac{3}{2} \sigma_{2}\right) \xi-\frac{3(1-\mu)(\xi+\mu)\left(2 \sigma_{1}-\sigma_{2}\right)}{2 \rho_{1}^{5}}-\frac{15(1-\mu)(\xi+\mu)\left(\sigma_{2}-\sigma_{1}\right) \eta^{2}}{2 \rho_{1}^{7}} \\
& +\frac{1}{c^{2}}\left[-3 \xi\left\{1-\frac{\mu(1-\mu)}{3}\right\}+\frac{1}{2} \xi\left(\xi^{2}+\eta^{2}\right)-\frac{3}{2}\left(\xi^{2}+\eta^{2}\right)\left\{\frac{(1-\mu)(\xi+\mu)}{\rho_{1}^{3}}+\frac{\mu(\xi-1+\mu)}{\rho_{2}^{3}}\right\}+3\left(\frac{1-\mu}{\rho_{1}}+\frac{\mu}{\rho_{2}}\right) \xi\right. \\
& +\frac{(1-\mu)^{2}(\xi+\mu)}{\rho_{1}^{4}}+\frac{\mu^{2}(\xi-1+\mu)}{\rho_{2}^{4}}+\mu(1-\mu)\left\{\frac{7}{2}\left(\frac{1}{\rho_{1}}-\frac{1}{\rho_{2}}\right)+\frac{7}{2} \xi\left(-\frac{(\xi+\mu)}{\rho_{1}^{3}}+\frac{(\xi-1+\mu)}{\rho_{2}^{3}}\right)\right. \\
& \left.\left.+\frac{3}{2} \eta^{2}\left(\frac{\mu(\xi+\mu)}{\rho_{1}^{5}}+\frac{(1-\mu)(\xi-1+\mu)}{\rho_{2}^{5}}\right)+\frac{(\xi+\mu)}{\rho_{1}^{3} \rho_{2}}+\frac{(\xi-1+\mu)}{\rho_{1} \rho_{2}^{3}}-\frac{(3 \mu-2)(\xi+\mu)}{2 \rho_{1}^{3}}-\frac{(1-3 \mu)(\xi-1+\mu)}{2 \rho_{2}^{3}}\right\}\right]=0
\end{aligned}
$$

and

$$
W_{\eta}=\eta F,
$$

with

$$
\begin{aligned}
& F=\left(1-\frac{1-\mu}{\rho_{1}^{3}}-\frac{\mu}{\rho_{2}^{3}}\right)+\left(3 \sigma_{1}-\frac{3}{2} \sigma_{2}\right)+\frac{3(1-\mu)}{\rho_{1}^{5}}\left(\frac{3}{2} \sigma_{2}-2 \sigma_{1}\right)-\frac{15(1-\mu)\left(\sigma_{2}-\sigma_{1}\right) \eta^{2}}{2 \rho_{1}^{7}}+ \\
& \frac{1}{c^{2}}\left[-3\left(1-\frac{\mu(1-\mu)}{3}\right)+\frac{1}{2}\left(\xi^{2}+\eta^{2}\right)+3\left(\frac{1-\mu}{\rho_{1}}+\frac{\mu}{\rho_{2}}\right)-\frac{3}{2}\left(\xi^{2}+\eta^{2}\right)\left(\frac{1-\mu}{\rho_{1}^{3}}+\frac{\mu}{\rho_{2}^{3}}\right)+\left(\frac{(1-\mu)^{2}}{\rho_{1}^{4}}+\frac{\mu^{2}}{\rho_{2}^{4}}\right)\right. \\
& \left.+\mu(1-\mu)\left\{\frac{7}{2} \xi\left(-\frac{1}{\rho_{1}^{3}}+\frac{1}{\rho_{2}^{3}}\right)-\left(\frac{\mu}{\rho_{1}^{3}}+\frac{1-\mu}{\rho_{2}^{3}}\right)+\frac{3}{2} \eta^{2}\left(\frac{\mu}{\rho_{1}^{5}}+\frac{1-\mu}{\rho_{2}^{5}}\right)+\frac{1}{\rho_{1}^{3} \rho_{2}}+\frac{1}{\rho_{1} \rho_{2}^{3}}-\frac{(3 \mu-2)}{2 \rho_{1}^{3}}-\frac{(1-3 \mu)}{2 \rho_{2}^{3}}\right\}\right] .
\end{aligned}
$$


In order to find the collinear points, we put $\eta=0$ in equation (5). Their abscissae are the roots of the equation

$$
\begin{aligned}
& f(\xi)=\xi-\frac{(1-\mu)(\xi+\mu)}{\rho_{1}^{3}}-\frac{\mu(\xi-1+\mu)}{\rho_{2}^{3}}+\left(3 \sigma_{1}-\frac{3}{2} \sigma_{2}\right) \xi-\frac{3(1-\mu)(\xi+\mu)\left(2 \sigma_{1}-\sigma_{2}\right)}{2 \rho_{1}^{5}} \\
& +\frac{1}{c^{2}}\left[-3 \xi\left(1-\frac{\mu(1-\mu)}{3}\right)+\frac{1}{2} \xi^{3}-\frac{3}{2} \xi^{2}\left\{\frac{(1-\mu)(\xi+\mu)}{\rho_{1}^{3}}+\frac{\mu(\xi-1+\mu)}{\rho_{2}^{3}}\right\}+3\left(\frac{1-\mu}{\rho_{1}}-\frac{\mu}{\rho_{2}}\right) \xi\right. \\
& +\frac{(1-\mu)^{2}(\xi+\mu)}{\rho_{1}^{4}}+\frac{\mu^{2}(\xi-1+\mu)}{\rho_{2}^{4}}+\mu(1-\mu)\left\{\frac{7}{2}\left(\frac{1}{\rho_{1}}-\frac{1}{\rho_{2}}\right)+\frac{7}{2} \xi\left(-\frac{(\xi+\mu)}{\rho_{1}^{3}}+\frac{(\xi-1+\mu)}{\rho_{2}^{3}}\right)\right. \\
& \left.\left.+\frac{\xi-1+\mu}{\rho_{1} \rho_{2}^{3}}+\frac{\xi+\mu}{\rho_{1}^{3} \rho_{2}}-\frac{(3 \mu-2)(\xi+\mu)}{2 \rho_{1}^{3}}-\frac{(1-3 \mu)(\xi-1+\mu)}{2 \rho_{2}^{3}}\right\}\right]=0
\end{aligned}
$$

with $\rho_{1}=|\xi+\mu|, \rho_{2}=|\xi-1+\mu|$.

To locate the collinear points on the $\xi$-axis, we divide the orbital plane into three parts: $\xi<\xi_{1}, \quad \xi_{1}<\xi<\xi_{2}$ and $\xi_{2}<\xi$ with respect to the primaries.

$$
m_{1}=1-\mu \quad m_{2}=\mu \quad \lambda_{1} \quad L_{1}
$$

(a)

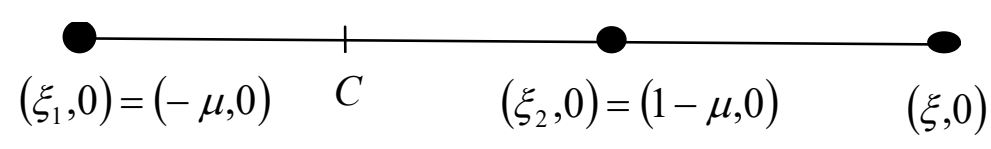

$$
m_{1}=1-\mu \quad L_{2} \quad \lambda_{2} \quad m_{2}=\mu
$$

(b)

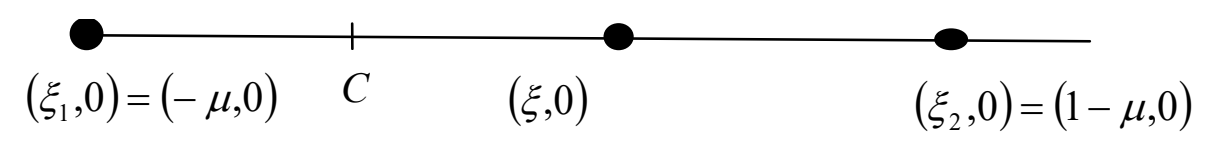

$$
\begin{array}{lll}
L_{3} & 1-\lambda_{3} \quad m_{1}=1-\mu & m_{2}=\mu
\end{array}
$$

(c)

$$
(\xi, 0) \quad\left(\xi_{1}, 0\right)=(-\mu, 0) \quad C \quad\left(\xi_{2}, 0\right)=(1-\mu, 0)
$$

Figure 1. Reference parameter for collinear Lagrangian points.

Case 1: Position of $L_{1}\left(\xi>\xi_{2}\right)$ (see Fig. 1 (a))

Let $\xi-\xi_{2}=\lambda_{1} ; \xi-\xi_{1}=1+\lambda_{1} \Rightarrow \xi=1+\lambda_{1}+\xi_{1}$; since the distance between the primaries is unity, i.e. $\xi_{2}-\xi_{1}=1 \Rightarrow \xi_{1}=-\mu$ and $\xi_{2}=1-\mu$ then

$$
\xi=1+\lambda_{1}-\mu ; \rho_{1}=1+\lambda_{1} ; \rho_{2}=\lambda_{1}
$$


Now substituting equation (7) in (6), we obtain

$$
\begin{aligned}
& \lambda^{9}+(-3 \mu+7) \lambda_{1}^{8}+\left(6 c^{2} \sigma_{1}-16 \mu+15-3 c^{2} \sigma_{2}+\mu^{2}+2 c^{2}\right) \lambda_{1}^{7}+\left(-6 c^{2} \sigma_{1} \mu+3 \mu c^{2} \sigma_{2}+30 c^{2} \sigma_{1}-15 c^{2} \sigma_{2}+\mu^{3}\right. \\
& \left.+10 c^{2}+3 \mu^{2}-2 \mu c^{2}+8-29 \mu\right) \lambda_{1}^{6}+\left(20 c^{2}-8 \mu c^{2}-24 c^{2} \sigma_{1} \mu+12 \mu c^{2} \sigma_{2}-13+60 c^{2} \sigma_{1}-16 \mu+4 \mu^{3}-30 c^{2} \sigma_{2}+4 \mu^{2}\right) \lambda_{1}^{5} \\
& +\left(15 \mu^{2}+18 c^{2}+2 \mu-12 \mu c^{2}+6 \mu^{3}+60 c^{2} \sigma_{1}-36 \mu c^{2} \sigma_{1}-30 c^{2} \sigma_{2}+18 \mu c^{2} \sigma_{2} \mu-21\right) \lambda_{1}^{4}+\left(6 c^{2}+2 \mu^{3}-3 \mu+34 \mu^{2}\right. \\
& \left.-6 \mu^{4}+30 c^{2} \sigma_{1}-12 \mu c^{2}-9-24 \mu c^{2} \sigma_{1}-15 c^{2} \sigma_{2}+12 \mu c^{2} \sigma_{2}\right) \lambda_{1}^{3}+\left(3 \mu-6 \mu^{4}-12 \mu c^{2}-19 \mu^{3}+33 \mu^{2}\right) \lambda_{1}^{2} \\
& +\left(13 \mu+13 \mu^{2}-8 \mu c^{2}-24 \mu^{3}\right) \lambda_{1}+\left(-6 \mu^{3}+\mu^{2}-2 \mu c^{2}+5 \mu\right)=0
\end{aligned}
$$

In the presence of triaxiality only, we have

$$
\begin{aligned}
& \left(3 \sigma_{2}-6 \sigma_{1}-2\right) \lambda_{1}^{7}+\left(6 \sigma_{1} \mu-3 \sigma_{2} \mu-30 \sigma_{1}+15 \sigma_{2}+2 \mu-10\right) \lambda_{1}^{6}+\left(30 \sigma_{2}+24 \sigma_{1} \mu-12 \sigma_{2} \mu+8 \mu-60 \sigma_{1}-20\right) \lambda_{1}^{5} \\
& +\left(-60 \sigma_{1}+36 \sigma_{1} \mu+12 \mu-18 \sigma_{2} \mu-18+30 \sigma_{2}\right) \lambda_{1}^{4}+\left(-12 \sigma_{2} \mu+12 \mu-30 \sigma_{1}+24 \sigma_{1} \mu+15 \sigma_{2}-6\right) \lambda_{1}^{3} \\
& +12 \mu \lambda_{1}^{2}+8 \mu \lambda_{1}+2 \mu=0
\end{aligned}
$$

Case 2: Position of $L_{2}\left(\xi_{1}<\xi<\xi_{2}\right)$ (see Fig. 1 (b))

$$
\text { Let } \xi_{2}-\xi=\lambda_{2} ; \xi-\xi_{1}=1-\lambda_{2} \Rightarrow \xi=1-\lambda_{2}-\mu ; \rho_{1}=1-\lambda_{2} ; \rho_{2}=\lambda_{2}
$$

Substituting equation (9) in (6), we obtain

$$
\begin{aligned}
& -\lambda_{2}{ }^{9}+(-3 \mu+7) \lambda_{2}^{8}+\left(16 \mu+3 c^{2} \sigma_{2}-2 c^{2}-\mu^{2}-6 c^{2} \sigma_{1}-15\right) \lambda_{2}{ }^{7}+\left(-2 \mu c^{2}+3 \mu^{2}+8+10 c^{2}+30 c^{2} \sigma_{1}+\mu^{3}-35 \mu\right. \\
& \left.+3 \mu c^{2} \sigma_{2}-6 c^{2} \sigma_{1} \mu-15 c^{2} \sigma_{2}\right) \lambda_{2}{ }^{6}+\left(10 \mu^{2}+8 \mu c^{2}+26 \mu+13-20 c^{2}-60 c^{2} \sigma_{1}+30 c^{2} \sigma_{2}-4 \mu^{3}-12 \mu c^{2} \sigma_{2}+24 c^{2} \sigma_{1} \mu\right) \lambda_{2}{ }^{5} \\
& +\left(-47 \mu^{2}+18 \mu^{3}+60 c^{2} \sigma_{1}+16 \mu-36 \mu c^{2} \sigma_{1}-8 \mu c^{2}+18 c^{2} \sigma_{2} \mu+18 c^{2}-30 c^{2} \sigma_{2}-21\right) \lambda_{2}{ }^{4}+\left(15 c^{2} \sigma_{2}-25 \mu-30 c^{2} \sigma_{1}\right. \\
& \left.+24 \mu c^{2} \sigma_{1}-12 \mu c^{2} \sigma_{2}-50 \mu^{3}-4 \mu c^{2}+9+6 \mu^{4}+66 \mu^{2}-6 c^{2}\right) \lambda_{2}{ }^{3}+\left(53 \mu^{3}-6 \mu^{4}-39 \mu^{2}+12 \mu c^{2}-3 \mu\right) \lambda_{2}{ }^{2} \\
& +\left(13 \mu-8 \mu c^{2}+9 \mu^{2}-24 \mu^{3}\right) \lambda_{2}+\left(-\mu^{2}-5 \mu+6 \mu^{3}+2 \mu c^{2}\right)=0
\end{aligned}
$$

In the presence of triaxiality only, we have

$$
\begin{aligned}
& \left(6 \sigma_{1}-3 \sigma_{2}+2\right) \lambda_{2}^{7}+\left(-10+15 \sigma_{2}+2 \mu-3 \sigma_{2} \mu+6 \sigma_{1} \mu-30 \sigma_{1}\right) \lambda_{2}^{6}+\left(60 \sigma_{1}+12 \sigma_{2} \mu-8 \mu-30 \sigma_{2}-24 \sigma_{1} \mu+20\right) \lambda_{2}^{5} \\
& +\left(36 \sigma_{1} \mu-60 \sigma_{1}-18 \sigma_{2} \mu+8 \mu+30 \sigma_{2}-18\right) \lambda_{2}^{4}+\left(-24 \sigma_{1} \mu+4 \mu+12 \sigma_{2} \mu+30 \sigma_{1}-15 \sigma_{2}+6\right) \lambda_{2}^{3} \\
& -12 \mu \lambda_{2}^{2}+8 \mu \lambda_{2}-2 \mu=0
\end{aligned}
$$

Case 3: Position of $L_{3}\left(\xi<\xi_{1}\right)$ (see Fig. 1 (b))

Let the distance of the point $L_{3}$ from the bigger primary be $1-\lambda_{3}$.

Since $\xi_{2}-\xi_{1}=1 \Rightarrow \xi_{1}-\xi=1-\lambda_{3} ; \xi_{2}-\xi=2-\lambda_{3}$ and

$$
\xi=\lambda_{3}-\mu-1 ; \rho_{1}=1-\lambda_{3} ; \rho_{2}=2-\lambda_{3}
$$


substituting equation (11) in (6), we obtain

$$
\begin{aligned}
& \lambda_{3}^{9}+(-3 \mu-11) \lambda_{3}^{8}+\left(47-3 c^{2} \sigma_{2}+6 c^{2} \sigma_{1}+32 \mu+2 c^{2}+\mu^{2}\right) \lambda_{3}^{7}+\left(27 c^{2} \sigma_{2}-6 c^{2} \sigma_{1} \mu-141 \mu-11 \mu^{2}-96-2 \mu c^{2}\right. \\
& \left.+3 \mu c^{2} \sigma_{2}-18 c^{2}+\mu^{3}-54 c^{2} \sigma_{1}\right) \lambda_{3}{ }^{6}+\left(204 c^{2} \sigma_{1}-8 \mu^{3}+48 c^{2} \sigma_{1} \mu+52 \mu^{2}-102 c^{2} \sigma_{2}+79+332 \mu-24 c^{2} \sigma_{2} \mu+68 c^{2}\right. \\
& \left.+16 \mu c^{2}\right) \lambda_{3}^{5}+\left(-420 c^{2} \sigma_{1}-156 \mu c^{2} \sigma_{1}+78 \mu c^{2} \sigma_{2}+26 \mu^{3}+41-138 c^{2}-440 \mu-143 \mu^{2}+210 c^{2} \sigma_{2}-52 \mu c^{2}\right) \lambda_{3}^{4}+\left(158 c^{2}\right. \\
& \left.+244 \mu^{2}+291 \mu+510 c^{2} \sigma_{1}-42 \mu^{3}+92 \mu c^{2}-145+264 c^{2} \sigma_{1} \mu-132 c^{2} \sigma_{2} \mu+6 \mu^{4}-255 c^{2} \sigma_{2}\right) \lambda_{3}^{3}+\left(49 \mu^{3}-96 c^{2}+180 c^{2} \sigma_{2}\right. \\
& \left.-360 c^{2} \sigma_{1}-96 \mu c^{2}+120-15 \mu-30 \mu^{4}-259 \mu^{2}+126 c^{2} \sigma_{2} \mu-252 c^{2} \sigma_{1} \mu\right) \lambda_{3}^{2}+\left(-36-60 c^{2} \sigma_{2}+56 \mu c^{2}+120 c^{2} \sigma_{1}\right. \\
& \left.-101 \mu+48 \mu^{4}-52 \mu^{3}-72 c^{2} \sigma_{2} \mu+24 c^{2}+163 \mu^{2}+144 c^{2} \sigma_{1} \mu\right) \lambda_{3}+\left(45 \mu-47 \mu^{2}-24 \mu^{4}-48 c^{2} \sigma_{1} \mu+26 \mu^{3}\right. \\
& \left.24 c^{2} \sigma_{2} \mu-14 \mu c^{2}\right)=0
\end{aligned}
$$

In the presence of triaxiality only, we have

$$
\begin{aligned}
& \left(-6 \sigma_{1}+3 \sigma_{2}-2\right) \lambda_{3}^{7}+\left(2 \mu+54 \sigma_{1}+6 \sigma_{1} \mu-3 \sigma_{2} \mu-27 \sigma_{2}+18\right) \lambda_{3}^{6}+\left(-204 \sigma_{1}-16 \mu+102 \sigma_{2}-48 \sigma_{1} \mu+24 \sigma_{2} \mu-68\right) \lambda_{3}^{5} \\
& +\left(-78 \sigma_{2} \mu+420 \sigma_{1}-210 \sigma_{2}+156 \sigma_{1} \mu+52 \mu+138\right) \lambda_{3}^{4}+\left(-264 \sigma_{1} \mu+132 \sigma_{2} \mu-510 \sigma_{1}-92 \mu+255 \sigma_{2}-158\right) \lambda_{3}^{3} \\
& +\left(96 \mu+252 \sigma_{1} \mu-126 \sigma_{2} \mu-180 \sigma_{2}+360 \sigma_{1}+96\right) \lambda_{3}^{2}+\left(-56 \mu+72 \sigma_{2} \mu-144 \sigma_{1} \mu+60 \sigma_{2}-120 \sigma_{1}-24\right) \lambda_{3} \\
& +14 \mu+48 \sigma_{1} \mu-24 \sigma_{2} \mu=0
\end{aligned}
$$

It is noticed that in each case there exists only one physically reasonable root.

\section{Stability of collinear points}

We examine the stability of an equilibrium configuration that is its ability to restrain the body motion in its vicinity. To do so we displace the infinitesimal body a little from an equilibrium point with small velocity. If its motion is rapid departure from vicinity of the point, we call such a position of equilibrium an unstable one. If the body oscillates about the point, it is said to be a stable position.

As Singh and Bello $[12,13]$ to study the stability of the collinear points we linearize the equations of motion (1) by using Taylor's expansion and neglecting second and higher order terms of $\sigma_{1}, \sigma_{2}, \frac{1}{c^{2}}$ and also their products, the characteristic equation is given by

$$
\left(P_{1} q_{2}-P_{2} q_{1}\right) \omega^{4}+\left(P_{1} q_{6}+P_{5} q_{2}+P_{3} q_{4}-P_{6} q_{1}-P_{2} q_{5}-P_{4} q_{3}\right) \omega^{2}+P_{5} q_{6}-P_{6} q_{5}=0
$$

where,

$$
\begin{aligned}
& P_{1}=1+W_{\dot{\xi} \xi}^{0}, P_{2}=W_{\dot{\eta} \xi}^{0}, P_{3}=W_{\xi \xi}^{0}-W_{\xi \xi}^{0}=0, P_{4}=W_{\eta \dot{\xi}}^{0}-2 n-W_{\xi \dot{\eta}}^{0}, \\
& P_{5}=-W_{\xi \xi}^{0}, P_{6}=-W_{\xi \eta}^{0}, q_{1}=W_{\xi \dot{\xi}}^{0}, q_{2}=1+W_{\dot{\eta} \eta}^{0}, q_{3}=2 n+W_{\xi \dot{\eta}}^{0}-W_{\eta \dot{\xi}}^{0}, \\
& q_{4}=W_{\eta \dot{\eta}}^{0}-W_{\eta \dot{\eta}}^{0}=0, q_{5}=-W_{\xi \xi}^{0}, q_{6}=-W_{\eta \eta}^{0} .
\end{aligned}
$$

The second order partial derivative of $W$ are denoted by subscripts. The superscripts 0 indicates that the derivative is to be evaluated at the collinear equilibrium points $\left(\xi_{0}, \eta_{0}\right)$ under consideration. 
The second order derivatives are

$W_{\xi \xi}=1+\frac{3(1-\mu)(\xi+\mu)^{2}}{\rho_{1}^{5}}-\frac{(1-\mu)}{\rho_{1}^{3}}+\frac{3 \mu(\xi-1+\mu)^{2}}{\rho_{2}^{5}}-\frac{\mu}{\rho_{2}^{3}}+\left(3 \sigma_{1}-\frac{3}{2} \sigma_{2}\right)+\frac{15(1-\mu)\left(2 \sigma_{1}-\sigma_{2}\right)(\xi+\mu)^{2}}{2 \rho_{1}^{7}}$ $-\frac{3(1-\mu)\left(2 \sigma_{1}-\sigma_{2}\right)}{2 \rho_{1}^{5}}+\frac{35(3-3 \mu)(\xi+\mu)^{2} \eta^{2}}{2 \rho_{1}^{9}}-\frac{5(3-3 \mu)\left(\sigma_{2}-\sigma_{1}\right) \eta^{2}}{2 \rho_{1}^{7}}+\frac{1}{c^{2}}\left[(\mu(1-\mu)-3)+(\dot{\eta}+\xi)^{2}\right.$

$\frac{1}{2}\left(\dot{\xi}^{2}+\dot{\eta}^{2}\right)+\xi \dot{\eta}-\eta \dot{\xi}+\frac{1}{2}\left(\xi^{2}+\eta^{2}\right)+\left(\frac{9(1-\mu)(\xi+\mu)^{2}}{2 \rho_{1}^{5}}-\frac{3(1-\mu)}{2 \rho_{1}^{3}}-\frac{3 \mu}{2 \rho_{2}^{3}}+\frac{9 \mu(\xi-1+\mu)^{2}}{2 \rho_{2}^{5}}\right)\left(\dot{\xi}^{2}+\dot{\eta}^{2}\right.$

$\left.+2 \xi \dot{\eta}-2 \eta \dot{\xi}+\xi^{2}+\eta^{2}\right)+2\left(\frac{-3(1-\mu)(\xi+\mu)}{\rho_{1}^{3}}-\frac{3 \mu(\xi-1+\mu)}{\rho_{2}^{3}}\right)(\dot{\eta}+\xi)+\frac{3(1-\mu)}{\rho_{1}}+\frac{3 \mu}{\rho_{2}}-\frac{4(1-\mu)^{2}(\xi+\mu)^{2}}{\rho_{1}^{6}}$

$+\frac{(1-\mu)^{2}}{\rho_{1}^{4}}-\frac{4 \mu^{2}(\xi-1+\mu)^{2}}{\rho_{2}^{6}}+\frac{\mu^{2}}{\rho_{2}^{4}}+\mu(1-\mu)\left\{\left(-\frac{7(\xi+\mu)}{\rho_{1}^{3}}+\frac{7(\xi-1+\mu)}{\rho_{2}^{3}}\right)+\left(\frac{3(\xi+\mu)^{2}}{\rho_{1}^{5}}-\frac{1}{\rho_{1}^{3}}+\frac{1}{\rho_{2}^{3}}\right.\right.$

$\left.-\frac{3(\xi-1+\mu)^{2}}{\rho_{2}^{5}}\right)\left(4 \dot{\eta}+\frac{7}{2} \xi\right)-\frac{\eta^{2}}{2}\left(\frac{15 \mu(\xi+\mu)^{2}}{\rho_{1}^{7}}-\frac{3 \mu}{\rho_{1}^{5}}+\frac{15(1-\mu)(\xi-1+\mu)^{2}}{\rho_{2}^{7}}-\frac{3(1-\mu)}{\rho_{2}^{5}}\right)-\frac{3(\xi+\mu)^{2}}{\rho_{1}^{5} \rho_{2}}$

$-\frac{2(\xi+\mu)(\xi-1+\mu)}{\rho_{1}^{3} \rho_{2}^{3}}+\frac{1}{\rho_{1}^{3} \rho_{2}}-\frac{3(\xi-1+\mu)^{2}}{\rho_{1} \rho_{2}^{5}}+\frac{1}{\rho_{1} \rho_{2}^{3}}+\frac{3(3 \mu-2)(\xi+\mu)^{2}}{2 \rho_{1}^{5}}-\frac{(3 \mu-2)}{2 \rho_{1}^{3}}-\frac{(1-3 \mu)}{2 \rho_{2}^{3}}$

$\left.\left.+\frac{3(1-3 \mu)(\xi-1+\mu)^{2}}{2 \rho_{2}^{5}}\right\}\right]$

$W_{\eta \eta}=1+\frac{3(1-\mu) \eta^{2}}{\rho_{1}^{5}}-\frac{(1-\mu)}{\rho_{1}^{3}}+\frac{3 \mu \eta^{2}}{\rho_{2}^{5}}-\frac{\mu}{\rho_{2}^{3}}+\left(3 \sigma_{1}-\frac{3}{2} \sigma_{2}\right)+\frac{15(1-\mu)\left(2 \sigma_{1}-\sigma_{2}\right) \eta^{2}}{2 \rho_{1}^{7}}-\frac{3(1-\mu)\left(3 \sigma_{2}-4 \sigma_{1}\right)}{2 \rho_{1}^{5}}$

$-\frac{25(3-3 \mu)\left(\sigma_{2}-\sigma_{1}\right) \eta^{2}}{2 \rho_{1}^{7}}+\frac{35(3-3 \mu)\left(\sigma_{2}-\sigma_{1}\right) \eta^{4}}{2 \rho_{1}^{9}}+\frac{1}{c^{2}}\left[(\mu(1-\mu)-3)+(\eta-\dot{\xi})^{2}+\frac{3}{4}\left(\dot{\xi}^{2}+\dot{\eta}^{2}\right)+\frac{9}{4}(\xi \dot{\eta}-\eta \dot{\xi})\right.$

$+\frac{3}{2}\left(\xi^{2}+\eta^{2}\right)+\left(\frac{9(1-\mu) \eta^{2}}{2 \rho_{1}^{5}}-\frac{3(1-\mu)}{2 \rho_{1}^{3}}-\frac{3 \mu}{2 \rho_{2}^{3}}+\frac{9 \mu \eta^{2}}{2 \rho_{2}^{5}}\right)\left(\dot{\xi}^{2}+\dot{\eta}^{2}+2 \xi \dot{\eta}-2 \eta \dot{\xi}+\xi^{2}+\eta^{2}\right)$

$+2\left(\frac{-3(1-\mu) \eta}{\rho_{1}^{3}}-\frac{3 \mu \eta}{\rho_{2}^{3}}\right)(\eta-\dot{\xi})+\frac{3(1-\mu)}{\rho_{1}}+\frac{3 \mu}{\rho_{2}}-\frac{4(1-\mu)^{2} \eta^{2}}{\rho_{1}^{6}}+\frac{(1-\mu)^{2}}{\rho_{1}^{4}}-\frac{4 \mu^{2} \eta^{2}}{\rho_{2}^{6}}+\frac{\mu^{2}}{\rho_{2}^{4}}$

$+\mu(1-\mu)\left\{\left(\frac{3 \eta^{2}}{\rho_{1}^{5}}-\frac{1}{\rho_{1}^{3}}+\frac{1}{\rho_{2}^{3}}-\frac{3 \eta^{2}}{\rho_{2}^{5}}\right)\left(4 \dot{\eta}+\frac{7}{2} \xi\right)-\frac{\mu}{\rho_{1}^{3}}-\frac{(1-\mu)}{\rho_{2}^{3}}+6 \eta^{2}\left(\frac{\mu}{\rho_{1}^{5}}+\frac{(1-\mu)}{\rho_{2}^{5}}\right)\right.$

$-\frac{\eta^{2}}{2}\left(\frac{15 \mu \eta^{2}}{\rho_{1}^{7}}-\frac{3 \mu}{\rho_{1}^{5}}+\frac{15(1-\mu) \eta^{2}}{\rho_{2}^{7}}-\frac{3(1-\mu)}{\rho_{2}^{5}}\right)-\frac{3 \eta^{2}}{\rho_{1}^{5} \rho_{2}}-\frac{2 \eta^{2}}{\rho_{1}^{3} \rho_{2}^{3}}+\frac{1}{\rho_{1}^{3} \rho_{2}}-\frac{3 \eta^{2}}{\rho_{1} \rho_{2}^{5}}+\frac{1}{\rho_{1} \rho_{2}^{3}}$

$\left.\left.+\frac{3(3 \mu-2) \eta^{2}}{2 \rho_{1}^{5}}-\frac{(3 \mu-2)}{2 \rho_{1}^{3}}-\frac{(1-3 \mu)}{2 \rho_{2}^{3}}+\frac{3(1-3 \mu) \eta^{2}}{2 \rho_{2}^{5}}\right\}\right]$

$W_{\xi \eta}=\frac{3(1-\mu)(\xi+\mu) \eta}{\rho_{1}^{5}}+\frac{3 \mu(\xi-1+\mu) \eta}{\rho_{2}^{5}}+\frac{15(1-\mu)\left(2 \sigma_{1}-\sigma_{2}\right)(\xi+\mu) \eta}{2 \rho_{1}^{7}}-\frac{5(3-3 \mu)\left(\sigma_{2}-\sigma_{1}\right)(\xi+\mu) \eta}{\rho_{1}^{7}}$

$+\frac{35(3-3 \mu)\left(\sigma_{2}-\sigma_{1}\right)(\xi+\mu) \eta^{3}}{2 \rho_{1}^{9}}+\frac{1}{c^{2}}\left[(\dot{\eta}+\xi)(\eta-\dot{\xi})+\left(\frac{9(1-\mu)(\xi+\mu) \eta}{2 \rho_{1}^{5}}+\frac{9 \mu(\xi-1+\mu) \eta}{2 \rho_{2}^{5}}\right)\left(\dot{\xi}^{2}+\dot{\eta}^{2}\right.\right.$

$\left.+2 \xi \dot{\eta}-2 \eta \dot{\xi}+\xi^{2}+\eta^{2}\right)+\left(-\frac{3(1-\mu) \eta}{\rho_{1}^{3}}-\frac{3 \mu \eta}{\rho_{2}^{3}}\right)(\dot{\eta}+\xi)+\left(-\frac{3(1-\mu)(\xi+\mu)}{\rho_{1}^{3}}-\frac{3 \mu(\xi-1+\mu)}{\rho_{2}^{3}}\right)(\eta-\dot{\xi})$

$-\frac{4(1-\mu)^{2}(\xi+\mu) \eta}{\rho_{1}^{6}}-\frac{4 \mu^{2}(\xi-1+\mu) \eta}{\rho_{2}^{6}}+\mu(1-\mu)\left\{\frac{7 \eta}{2 \rho_{2}^{3}}-\frac{7 \eta}{2 \rho_{2}^{3}}+\left(4 \dot{\eta}+\frac{7}{2} \xi\right)\left(\frac{3(\xi+\mu) \eta}{\rho_{1}^{5}}-\frac{3(\xi-1+\mu) \eta}{\rho_{2}^{5}}\right)\right.$

$+\eta\left(\frac{3 \mu(\xi+\mu)}{\rho_{1}^{5}}+\frac{3(1-\mu)(\xi-1+\mu)}{\rho_{2}^{5}}\right)-\frac{\eta^{2}}{2}\left(\frac{15 \mu(\xi+\mu) \eta}{\rho_{1}^{7}}+\frac{15(1-\mu)(\xi-1+\mu) \eta}{\rho_{2}^{7}}\right)-\frac{3(\xi+\mu) \eta}{\rho_{1}^{5} \rho_{2}}-\frac{(\xi-1+\mu) \eta}{\rho_{1}^{3} \rho_{2}^{3}}$

$\left.\left.-\frac{(\xi+\mu) \eta}{\rho_{1}^{3} \rho_{2}^{3}}-\frac{3(\xi-1+\mu) \eta}{\rho_{1} \rho_{2}^{5}}+\frac{3(3 \mu-2)(\xi+\mu) \eta}{2 \rho_{1}^{5}}+\frac{3(1-3 \mu)(\xi-1+\mu) \eta}{2 \rho_{2}^{5}}\right\}\right]=0$ 


$$
\begin{aligned}
& W_{\dot{\xi} \xi}= \frac{1}{c^{2}}\left[(\dot{\xi}-\eta)^{2}+\frac{1}{2}\left(\dot{\xi}^{2}+\dot{\eta}^{2}\right)+(\xi \dot{\eta}-\eta \dot{\xi})+\frac{1}{2}\left(\xi^{2}+\eta^{2}\right)+\frac{3(1-\mu)}{\rho_{1}}+\frac{3 \mu}{\rho_{2}}\right] \\
& W_{\dot{\eta} \dot{\eta}}= \frac{1}{c^{2}}\left[(\dot{\eta}+\xi)^{2}+\frac{1}{2}(\dot{\xi}+\dot{\eta})^{2}+(\xi \dot{\eta}-\eta \dot{\xi})+\frac{1}{2}\left(\xi^{2}+\eta^{2}\right)+\frac{3(1-\mu)}{\rho_{1}}+\frac{3 \mu}{\rho_{2}}\right] \\
& W_{\dot{\eta} \dot{\xi}}=\frac{1}{c^{2}}[(\dot{\xi}-\eta)(\dot{\eta}+\xi)] \\
& W_{\xi \dot{\xi}}=\frac{1}{c^{2}}\left[(\dot{\eta}+\xi)(\dot{\xi}-\eta)-3(\dot{\xi}-\eta)\left(\frac{(1-\mu)(\xi+\mu)}{\rho_{1}^{3}}+\frac{\mu(\xi-1+\mu)}{\rho_{2}^{3}}\right)\right] \\
& W_{\xi \dot{\eta}}=\frac{1}{c^{2}}\left[\frac{1}{2}(\dot{\eta}+2 \xi) \xi+\frac{1}{2} \xi \dot{\eta}-\frac{1}{2} \dot{\xi} \eta+\frac{1}{2} \xi^{2}+\frac{1}{2} \eta^{2}\right. \\
&-\frac{3}{16}\left(\frac{(1-\mu)(\xi+\mu)}{\rho_{1}^{3}}+\frac{\mu(\xi+\mu-1)}{\rho_{2}^{3}}\right)\left(2 \dot{\eta}+2 \xi+4 \mu(1-\mu)\left(\frac{1}{\rho_{1}}-\frac{1}{\rho_{2}}\right)\right) \\
&\left.+\frac{3}{16}\left(\frac{1-\mu}{\rho_{1}^{3}}+\frac{\mu}{\rho_{2}}\right)\left(2+4 \mu(1-\mu)\left(\frac{\xi+\mu-1}{\rho_{2}^{3}}-\frac{\xi+\mu}{\rho_{1}^{3}}\right)\right)\right] \\
&\left.W_{\eta \dot{\xi}}=\frac{1}{c^{2}}\left[-\frac{1}{2}(2 \eta-\dot{\xi}) \eta-\frac{1}{\rho_{1}^{3}}+\frac{1}{\rho_{2}^{3}}\right)\right] \\
&\left.\left(\xi \dot{\eta}-\eta \dot{\xi}+\xi^{2}+\eta^{2}\right)-\frac{3}{8} \eta\left(\frac{1-\mu}{\rho_{1}^{3}}+\frac{\mu}{\rho_{2}^{3}}\right)(\dot{\xi}-\eta)-\frac{3}{8}\left(\frac{1-\mu}{\rho_{1}}+\frac{\mu}{\rho_{2}}\right)\right]
\end{aligned}
$$

In order to study the stability of the collinear points we have to study the motion in the proximity of these points, hence in this case (14a)-(23a) can be written as:

$$
\begin{aligned}
& W_{\xi \xi}^{0}=1+\frac{3(1-\mu)(\xi+\mu)^{2}}{\rho_{1}^{5}}-\frac{(1-\mu)}{\rho_{1}^{3}}+\frac{3 \mu(\xi-1+\mu)^{2}}{\rho_{2}^{5}}-\frac{\mu}{\rho_{2}^{3}}+\left(3 \sigma_{1}-\frac{3}{2} \sigma_{2}\right)+\frac{15(1-\mu)\left(2 \sigma_{1}-\sigma_{2}\right)(\xi+\mu)^{2}}{2 \rho_{1}^{7}} \\
& -\frac{3(1-\mu)\left(2 \sigma_{1}-\sigma_{2}\right)}{2 \rho_{1}^{5}}+\frac{1}{c^{2}}\left[(\mu(1-\mu)-3)+\frac{3}{2} \xi^{2}+\left(\frac{9(1-\mu)(\xi+\mu)^{2}}{2 \rho_{1}^{5}}-\frac{3(1-\mu)}{2 \rho_{1}^{3}}-\frac{3 \mu}{2 \rho_{2}^{3}}+\frac{9 \mu(\xi-1+\mu)^{2}}{2 \rho_{2}^{5}}\right) \xi^{2}\right. \\
& +\left(-\frac{3(1-\mu)(\xi+\mu)}{\rho_{1}^{3}}-\frac{3 \mu(\xi-1+\mu)}{\rho_{2}^{3}}\right) \xi+\frac{3(1-\mu)}{\rho_{1}}+\frac{3 \mu}{\rho_{2}}-\frac{4(1-\mu)^{2}(\xi+\mu)^{2}}{\rho_{1}^{6}}+\frac{(1-\mu)^{2}}{\rho_{1}^{4}}-\frac{4 \mu^{2}(\xi-1+\mu)^{2}}{\rho_{2}^{6}} \\
& +\frac{\mu^{2}}{\rho_{2}^{4}}+\mu(1-\mu)\left\{-\frac{7(\xi+\mu)}{\rho_{1}^{3}}+\frac{7(\xi-1+\mu)}{\rho_{2}^{3}}+\frac{7}{2}\left(\frac{3(\xi+\mu)^{2}}{\rho_{1}^{5}}-\frac{1}{\rho_{1}^{3}}+\frac{1}{\rho_{2}^{3}}-\frac{3(\xi-1+\mu)^{2}}{\rho_{2}^{5}}\right) \xi-\frac{3(\xi+\mu)^{2}}{\rho_{1}^{5} \rho_{2}}\right. \\
& -\frac{2(\xi+\mu)(\xi-1+\mu)}{\rho_{1}^{3} \rho_{2}^{3}}+\frac{1}{\rho_{1}^{3} \rho_{2}}-\frac{3(\xi-1+\mu)^{2}}{\rho_{1} \rho_{2}^{5}}+\frac{1}{\rho_{1} \rho_{2}^{3}}+\frac{3(3 \mu-2)(\xi+\mu)^{2}}{2 \rho_{1}^{5}}-\frac{(3 \mu-2)}{2 \rho_{1}^{3}}+\frac{3(1-3 \mu)(\xi-1+\mu)^{2}}{2 \rho_{2}^{5}} \\
& \left.\left.-\frac{(1-3 \mu)}{2 \rho_{2}^{3}}\right\}\right] \\
& W_{\eta \eta}^{0}=1-\frac{(1-\mu)}{\rho_{1}^{3}}-\frac{\mu}{\rho_{2}^{3}}+\left(3 \sigma_{1}-\frac{3}{2} \sigma_{2}\right)+\frac{3(1-\mu)\left(3 \sigma_{2}-4 \sigma_{1}\right)}{2 \rho_{1}^{5}}+\frac{1}{c^{2}}\left[\mu(1-\mu)-3+\frac{3}{2} \xi^{2}+\left(-\frac{3(1-\mu)}{2 \rho_{1}^{3}}-\frac{3 \mu}{2 \rho_{2}^{3}}\right) \xi^{2}\right. \\
& +\frac{3(1-\mu)}{\rho_{1}}+\frac{3 \mu}{\rho_{2}}+\frac{(1-\mu)^{2}}{\rho_{1}^{4}}+\frac{\mu^{2}}{\rho_{2}^{4}}+\mu(1-\mu)\left\{\frac{7}{2}\left(-\frac{1}{\rho_{1}^{3}}+\frac{1}{\rho_{2}^{3}}\right) \xi-\frac{\mu}{\rho_{1}^{3}}-\frac{(1-\mu)}{\rho_{2}^{3}}+\frac{1}{\rho_{1}^{3} \rho_{2}}+\frac{1}{\rho_{1} \rho_{2}^{3}}-\frac{(3 \mu-2)}{2 \rho_{1}^{3}}\right. \\
& \left.\left.-\frac{(1-3 \mu)}{2 \rho_{2}^{3}}\right\}\right]
\end{aligned}
$$




$$
\begin{gathered}
W_{\xi \eta}^{0}=0 \\
W_{\dot{\xi} \xi}^{0}=\frac{1}{c^{2}}\left[\frac{1}{2} \xi^{2}+\frac{3(1-\mu)}{\rho_{1}}+\frac{3 \mu}{\rho_{2}}\right] \\
W_{\dot{\eta} \dot{\eta}}^{0}=\frac{1}{c^{2}}\left[\frac{3}{2} \xi^{2}+\frac{3(1-\mu)}{\rho_{1}}+\frac{3 \mu}{\rho_{2}}\right] \\
W_{\dot{\eta} \xi}^{0}=0 \\
W_{\xi \dot{\xi}}^{0}=0 \\
W_{\eta \dot{\eta}}^{0}=0 \\
W_{\xi \dot{\eta}}^{0}=\frac{1}{c^{2}}\left[\frac{3}{2} \xi^{2}-\frac{3}{16}\left(\frac{(1-\mu)(\xi+\mu)}{\rho_{1}^{3}}+\frac{\mu(\xi+\mu-1)}{\rho_{2}^{3}}\right)\left(2 \xi+4 \mu(1-\mu)\left(\frac{1}{\rho_{1}}-\frac{1}{\rho_{2}}\right)\right.\right. \\
\left.+\frac{3}{16}\left(\frac{1-\mu}{\rho_{1}}+\frac{\mu}{\rho_{2}}\right)\left(2+4 \mu(1-\mu)\left(\frac{\xi+\mu-1}{\rho_{2}^{3}}-\frac{\xi+\mu}{\rho_{1}^{3}}\right)\right)\right] \\
W_{\eta \dot{\xi}}^{0}=\frac{1}{c^{2}}\left[-\frac{1}{2} \xi^{2}-\frac{3}{8}\left(\frac{1-\mu}{\rho_{1}}+\frac{\mu}{\rho_{2}}\right)\right]
\end{gathered}
$$

Now we will show the discriminant $\Delta$ of (13) is positive at the collinear points $L_{i}(i=1,2,3)$.

It is noticed that

$$
K=-4\left(p_{1} q_{2}-p_{2} q_{1}\right)\left(p_{5} q_{6}-p_{6} q_{5}\right)>0
$$

as shown below $K$ can also be written as

$$
K=-4 W_{\xi \xi}^{0} W_{\eta \eta}^{0}\left(1+W_{\dot{\xi} \xi}^{0}\right)\left(1+W_{\dot{\eta} \dot{\eta}}^{0}\right)
$$

From $(17 \mathrm{~b})$ and $(18 \mathrm{~b})$ it is clear that $\left(1+W_{\dot{\xi} \xi}^{0}\right)>0$ and $\left(1+W_{\dot{\eta} \dot{\eta}}^{0}\right)>0$

Now we will study the signs of $W_{\xi \xi}^{0}$ and $W_{\eta \eta}^{0}$ at the collinear points $L_{i}(i=1,2,3)$

Firstly we will do this at $L_{1}$, since the coordinate of this point is $\left(1+\lambda_{1}-\mu, 0\right)$, then $r_{1}=1+\lambda_{1}$ and $r_{2}=\lambda_{1}$ where $0<\lambda_{1}<<1$, hence we can write $W_{\xi \xi}^{0}$ and $W_{\eta \eta}^{0}$ as a function in $\lambda_{1}$ say $g\left(\lambda_{1}\right)$ and $h\left(\lambda_{1}\right)$, respectively. Therefore, in the case from $(14 \mathrm{~b}), g\left(\lambda_{1}\right) \cong g\left(0^{+}\right)=-\infty$ and from (15b), $h\left(\lambda_{1}\right) \cong h\left(0^{+}\right)=+\infty$, hence $W_{\xi \xi}^{0}<0$ and $W_{\eta \eta}^{0}>0$ in which $W_{\xi \xi}^{0} W_{\eta \eta}^{0}<0$ and consequently $K>0$. Hence, the discriminant $\Delta$ of the characteristic equation (13) is positive, and the characteristic roots can be written as $\omega_{1,2}= \pm s, \omega_{3,4}= \pm i s^{\prime}$. where $s$ and $s^{\prime}$ are real.

Thus, $\omega_{1,2}$ are real and $\omega_{3,4}$ are pure imaginary, hence the motion around the collinear point $L_{1}$ is unbounded and the solution is unstable. Similarly, it can be shown that the points $L_{2}, L_{3}$ are also unstable. 


\section{Numerical results (Earth-Moon System)}

\subsection{Locations}

For the effect of triaxiality factor on the positions of the collinear points of the Earth-Moon systems, we take five different cases of different set of semi-axes in $\mathrm{Km}$. $(a, b, h)$ of the bigger primary i.e. $(6400,6400,6400),(6400,6390,6380),(6400,6380,6360),(6400,6370,6340)$ and $(6400,6360,6320)$. We have calculated the locations of the three collinear libration points in all the five cases by using the method in the above mentioned analysis. The second entries in table 1 correspond to the positions of collinear points in the presence of triaxiality only. Some of the data has been borrowed from Sharma and SubbaRao [16] and Dermott \& Murray [17].

Table 1. Collinear libration points (Earth-Moon system, $\mu=0.0121314293, c=292624.8185, R=384000 \mathrm{~km})$.

\begin{tabular}{|l|l|l|l|l|l|l|}
\hline $\begin{array}{l}\text { Para- } \\
\text { meter }\end{array}$ & \multicolumn{1}{|c|}{ Classical } & \multicolumn{1}{|c|}{ Case 1 } & Case 2 & Case 3 & Case 4 & Case 5 \\
\hline$\sigma_{1}$ & & 0 & $3.466796875 \times 10^{-7}$ & $6.922743056 \times 10^{-7}$ & $10.36783854 \times 10^{-7}$ & $13.80208333 \times 10^{-7}$ \\
\hline$\sigma_{2}$ & & 0 & $1.732042101 \times 10^{-7}$ & $3.455946181 \times 10^{-7}$ & $5.171712240 \times 10^{-7}$ & $6.879340278 \times 10^{-7}$ \\
\hline$L_{1}$ & 1.155608482288 & 1.155608442400 & 1.155608376400 & 0.155608310600 & 1.155608245000 & 1.155608179500 \\
& & & 1.155608376400 & 0.155608310600 & 1.155608244900 & 1.155608179500 \\
\hline$L_{2}$ & 0.837009519196 & 0.837009426300 & 0.837009499800 & 0.837009573200 & 0.837009646300 & 0.837009719200 \\
& & & 0.837009499800 & 0.837009572000 & 0.837009646100 & 0.837009719000 \\
\hline$L_{3}$ & -1.005054850445 & -1.005054664478 & -1.005054667392 & -1.005054670299 & -1.005054673196 & -1.005054676084 \\
& & & -1.005054667389 & -1.005054670299 & -1.005054673196 & -1.005054676085 \\
\hline
\end{tabular}

\subsection{Stability at Point $L_{1}$ for the Earth-Moon System}

We numerically investigate the stability of the collinear equilibrium point $L_{1}$ for the EarthMoon System. For this, we compute the value of $\lambda_{1}$ using equation (8) and roots of characteristic equation (13) for varying the triaxiality parameters, and list them in table 2.

Table 2. Roots of the characteristic equation (13).

\begin{tabular}{|c|c|c|c|c|c|}
\hline & $\begin{array}{c}\text { Case 1 } \\
\sigma_{1}=\sigma_{2}=0\end{array}$ & $\begin{array}{c}\text { Case 2 } \\
\sigma_{1}=3.466796875 \times 10^{-7} \\
\sigma_{2}=1.732042101 \times 10^{-7}\end{array}$ & $\begin{array}{c}\text { Case 3 } \\
\sigma_{1}=6.922743056 \times 10^{-7} \\
\sigma_{2}=3.455946181 \times 10^{-7}\end{array}$ & $\begin{array}{c}\text { Case 4 } \\
\sigma_{1}=10.36783854 \times 10^{-7} \\
\sigma_{2}=5.171712240 \times 10^{-7}\end{array}$ & $\begin{array}{c}\text { Case 5 } \\
\sigma_{1}=13.80208333 \times 10^{-7} \\
\sigma_{2}=6.879340278 \times 10^{-7}\end{array}$ \\
\hline$\lambda_{1}$ & 0.1677398717 & 0.1677398057 & 0.1677397399 & 0.1677396743 & 0.1677396088 \\
\hline$p_{1}$ & 1.00000000003997 & 1.00000000003997 & 1.00000000003997 & 1.00000000003997 & 1.00000000003997 \\
\hline$p_{2}$ & 0 & 0 & 0 & 0 & 0 \\
\hline$p_{3}$ & 0 & 0 & 0 & 0 & 0 \\
\hline$p_{4}$ & -2.00000000000247 & -2.00000078023522 & -2.00000155843346 & -2.0000023345972 & -2.00000310872642 \\
\hline$p_{5}$ & -7.38159641408971 & -7.38160489282262 & -7.38161334680286 & -7.38162177603028 & -7.38163019001795 \\
\hline$p_{6}$ & 0 & 0 & 0 & 0 & 0 \\
\hline$q_{1}$ & 0 & 0 & 0 & 0 & 0 \\
\hline$q_{2}$ & 1.00000000005557 & 1.00000000005557 & 1.00000000005557 & 1.00000000005557 & 1.00000000005557 \\
\hline$q_{3}$ & 2.00000000000247 & 2.00000078023522 & 2.00000155843346 & 2.0000023345972 & 2.00000310872642 \\
\hline$q_{4}$ & 0 & 0 & 0 & 0 & 0 \\
\hline$q_{5}$ & 0 & 0 & 0 & 0 & 0 \\
\hline$q_{6}$ & 2.19079820705729 & 2.19080115787436 & 2.19080409992215 & 2.19080703320059 & 2.00000310872642 \\
\hline$\omega_{1,2}$ & \pm 2.15884883280832 & \pm 2.15885030197736 & \pm 2.15885176671498 & \pm 2.15885322702116 & \pm 2.15885468512126 \\
\hline$\omega_{3,4}$ & $\pm 1.86274799038583 i$ & $\pm 1.86274904700877 i$ & $\pm 1.86275010060262 i$ & $\pm 1.86275115116737 i$ & $\pm 1.862752200005353 i$ \\
\hline
\end{tabular}


It is clear from table 2 that for a specific set of parameters $\sigma_{1}, \sigma_{2}$ at least one of the roots among all has a positive real root. Thus we conclude that the equilibrium point $L_{1}$ is unstable.

We may examine the stability of other equilibrium points $L_{2}, L_{3}$ in the same manner as $L_{1}$. We will see that $L_{2}, L_{3}$ are also unstable.

\section{Discussion}

Equations (1)-(4) describe the motion of a third body under the influence of the triaxiality of the bigger primary. Equations (8), (10), (12) give respective positions of the collinear equilibrium points $L_{1}, L_{2}, L_{3}$ which are dependent of the relativistic terms and triaxiality coefficients while equations (8a), (10a), (12a), give their positions in the presence of triaxiality factor only. It is noticed in section 4 that the relativistic terms and triaxiality coefficients are unable to alter the instability characteristic behavior of the collinear points. This is confirmed numerically from section 5 by the presence of positive real roots of the characteristic equation (13) as shown in table 2. In the absence of triaxiality coefficients, it is observed from case 1 in table 1 that the positions of $L_{1}, L_{2}, L_{3}$ deviate from the classical ones. This indicates that the relativistic factor has an effect on these positions. It can also observed from cases 2-5 that the position of $L_{1}$ decreases with the increase of triaxiality coefficients while the position of $L_{2}$ and $L_{3}$ increases with the increase of triaxiality coefficients. This indicates that with the combined effect of relativistic and triaxiality, $L_{1}$ comes nearer to the primaries with the increase in triaxiality coefficient while $L_{2}$ and $L_{3}$ moves away from the more massive primary. By comparing first and second entries of each case of table 1 , it can be said that in the presence of triaxiality, the effect of relativistic factor does not show physically in most of the cases on the positions of $L_{1}$ and $L_{2}$ whereas it is shown on the positions of $L_{2}$ except for the case 2. It can be seen from table 1 that $L_{1}$ moves towards the origin from the classical positions due to relativistic effect. The triaxiality also shifts $L_{1}$ towards the origin from the classical position. The similar shift is also seen due to the joint effect. $L_{2}$ and $L_{3}$ move towards the origin from the classical position due to relativistic or triaxiality or joint effect.

\section{Conclusion}

A study of the effect of triaxiality on the locations and stability of collinear points is carried out. It is noticed that in spite of the presence of relativistic terms and triaxiality coefficient, the instability characteristic behavior of the collinear points remains unchanged. This is confirmed using numerical approach as it reveals the existence of at least a positive root. Consequently, the motion is unbounded and we conclude that the equilibrium points are unstable due to positive roots. A numerical survey of the Earth-Moon system showed that in the absence of triaxiality coefficients, the relativistic terms have an effect on the positions of $L_{1}, L_{2}$ and $L_{3}$. It is also noticed that $L_{1}$ comes nearer to the primaries with the increase in triaxiality coefficients while $L_{2}, L_{3}$ move away from the more massive primary with the increase in triaxiality coefficients. It is also found that for the Earth-Moon system, in the presence of triaxiality, the relativistic factor has no observable effect in most of the cases on the positions of $L_{1}$ and $L_{3}$ whereas it has a noticeable effect on the position of $L_{2}$ except for the case 2.

For the future work, the study of the effect of mass ratios on the locations and stability of collinear points is suggested. 


\section{References}

[1] V. Szebehely, Theory of orbits. The restricted problem of three- bodies, Academic Press, New York, 1967.

[2] V. A. Brumberg, Relativistic Celestial Mechanics, Nauka, Moscow, USSR, 1972.

[3] K.B. Bhatnagar, P.P. Hallan, Existence and stability of $L_{4,5}$ in the relativistic restricted threebody problem, Celest. Mech. Dyn. Astron. 69 (1998) 271-281.

[4] C.N. Douskos, E.A. Perdios, On the stability of equilibrium points in the relativistic restricted three-body problem, Celest. Mech. Dyn. Astron. 82 (2002) 317-321.

[5] M.K. Ahmed, F.A. Abd El-Salam, S.E. Abd El-Bar, On the stability of triangular Lagrangian equilibrium points in the relativistic restricted three-body problem, American Journal of Applied Sciences. 3 (2006) 1993-1998.

[6] O. Ragos et al., On the equilibrium points of the relativistic restricted three-body problem, Nonlinear Analysis. 47 (2001) 3413-3418.

[7] S.E. Abd El-Bar, F.A. Abd El-Salam, Computation of the locations of the libration points in the relativistic restricted three-body problem, American Journal of Applied sciences. 9(5) (2012) 659-665.

[8] S.E Abd El-Bar, F.A. Abd El-Salam, Analytical and semi analytical treatment of the collinear points in the photogravitational relativistic R3BP, Mathematical Problems in Engineering. 2013 (2013).

[9] F.A. Abd El-Salam, S.E. Abd El-Bar, On the triangular equilibrium points in the photogravitational relativistic restricted three-body problem, Astrophys. Space Sci. 349 (2014) 125-135.

[10] D.A. Katour, F.A. Abd El-Salam, M.O. Shaker, Relativistic restricted three-body problem with oblateness and photo-gravitational corrections to triangular equilibrium points, Astrophys. Space Sci. 351(1) (2014) 143-149.

[11] S.E. Abd El- Bar, F.A. Abd El Salam, M. Rasseem, Perturbed location of $L_{1}$ point in the photogravitational relativistic restricted three-body problem (R3BP) with oblate primaries, Canadian journal of physics. 93(3) (2015) 300-311.

[12] J. Singh, N. Bello, Motion around $\mathrm{L}_{4}$ in the perturbed relativistic R3BP, Astrophys. Space Sci. 351 (2014) 491-497.

[13] J. Singh, N. Bello, Effect of radiation pressure on the stability of $\mathrm{L}_{4,5}$ in the R3BP, Astrophys. Space Sci. 351 (2014) 483-490.

[14] J. Singh, N. Bello, Existence and stability of triangular points in the relativistic R3BP when the primaries are triaxial rigid bodies and sources of radiation, Journal of Astrophysics and Aerospace Technology. 4(1) (2016).

[15] S.W. McCuskey, Introduction to celestial mechanics, Addision-Wesley, 1963.

[16] R.K. Sharma, P.V.S. Rao, Collinear equilibria and their characteristic exponents in the restricted three-body problem when the primaries are oblate spheroids, Celest. Mech. 12 (1975) 189-201.

[17] C.D. Murray, S.F. Dermott, Solar System Dynamics, Cambridge University Press, 1999. 